\title{
Interpretación de la radiografía de tórax en los pacientes que ingresan en una Unidad de Corta Estancia. Relación con la estancia media
}

\author{
A. SUPERVÍA CAPARRÓS, M. D. ARANDA CÁRDENAS, J. ROYO LÁZARO', \\ J. MARTÍNEZ ORFILA' ${ }^{1}$, M. A. MÁRQUEZ LORENTE, E. SKAF PETERS, \\ J. GUTIÉRREZ CEBOLLADA
}

Servicio de Urgencias y Radiología ${ }^{1}$. Hospital Universitario del Mar. Barcelona

CHEST X-RAY INTERPRETATION ON PATIENTS ADMITTED IN A SHORT-STAY UNIT. RELATION WITH THE MEAN LENGTH OF STAY

\section{RESUMEN}

Introducción: Determinar la concordancia entre los médicos de un Servicio de Urgencias y un radiólogo en la interpretación de la radiografía de tórax (RxT) de pacientes ingresados en una unidad de estancia corta (UEC) y evaluar si ello influye en la estancia media.

Material y método: Se revisaron las historias clínicas de los pacientes ingresados en una UEC durante 4 meses. Se comparó la interpretación de la RxT realizada por el médico responsable del paciente en urgencias con la interpretación realizada por un radiólogo. Se determinó la estancia media y se analizó si la lectura de la RxT previa al ingreso por el radiólogo hubiera mejorado la estancia media.

Resultados: Se revisaron 260 historias clínicas. La concordancia total fue del $74,2 \%$, siendo mayor cuando el médico de urgencias era un residente mayor o un adjunto que cuando era un residente menor $(82 \%$ vs $66,4 \% ; \mathrm{p}<0,003)$. No se observaron diferencias según el horario en el que se realizó la lectura. Solo en 9 casos $(3,5 \%)$ se hubiera producido un cambio de diagnóstico y/o tratamiento. No se produjeron cambios en la estancia media de los pacientes según existiera concordancia o no, aunque en los casos en que se hubiera producido un cambio de diagnóstico y/o tratamiento se observó una tendencia a su aumento.

Conclusiones: La lectura de la RxT por un radiólogo previa al ingreso en una UEC no está justificada en todos los casos. Podría realizarse cuando el residente mayor o el adjunto de guardia tuvieran dudas en su interpretación. Una mayor supervisión de los residentes menores podría mejorar el rendimiento diagnóstico de la RxT.

PALABRAS CLAVE: Unidad de estancia corta. Radiografía de tórax. Concordancia. Estancia media.

\begin{abstract}
Introduction: To assess the agreement between physicians of the emergency department and a radiologist in the interpretation of chest roentgenograms in patients admitted to the short-stay unit, and to determine the effect of concordance on the mean length of stay.

Material and method: The medical records of patients admitted to the short-stay unit over a 4-month period were reviewed. The interpretation of the chest $X$-ray film made by the physician in charge of the patient at the emergency department with that made by the radiologist was compared. The mean length of stay was determined and it was analyzed if the reading of the radiograph made by the radiologist before admission would decrease the length of stay.

Results: A total of 260 medical records were reviewed. The overall degree of agreement was $74.2 \%$ but it was greater when the physician at the emergency department was a staff physician or a resident in the final period of training than a resident in the initial period of training $(82 \% \mathrm{vs}$ $66.4 \%, p<0.003)$. There were no statistically significant differences regarding the day hours at which readings were made. A change in the diagnosis and/or treatment of the patient would had occurred in only 9 cases $(3.5 \%)$. There were no changes in the mean length of stay according to the presence or absence of concordance, although there was a trend towards an increase in the length of stay for those cases in which diagnosis and/or treatment would had been different.

Conclusions: Readings of chest roentgenograms by a radiologist before admission to a short-stay unit does not appear to be justified for all patients, although it may be justified when a staff physician or a resident in the final period of training have doubts regarding interpretation of the radiographic images. A better supervision of residents in the initial period of training may contribute to improve the diagnostic reliability of chest $X$-ray films.
\end{abstract}

KEY WORDS: Short-stay observation unit. Chest radiographs. Agreement. Mean length of stay.

Supervía Caparrós A, Aranda Cárdenas MD, Royo Lázaro J, Martínez Orfila J, Márquez Lorente MA, Skaf Peters E, Gutiérrez Cebollada J. Interpretación de la radiografía de tórax en los pacientes que ingresan en una Unidad de Corta Estancia. Relación con la estancia media. An Med Interna (Madrid) 2005; 22: 409-412.

\section{INTRODUCCIÓN}

En los últimos años se ha incrementado de forma considerable la frecuentación de los Servicios de Urgencias, de forma que en muchas ocasiones se llega a una situación de colapso.
Una de las soluciones para poder atender este incremento de la demanda asistencial ha sido la creación de las Unidades de Estancia Corta (UEC) con el objetivo de reducir estancias en pacientes que no precisen de ingreso en una unidad especializada (1). Asimismo, para optimizar la eficiencia de las UEC 
es aconsejable establecer unos criterios de ingreso (1-3), por lo que es fundamental realizar un diagnóstico correcto previo al ingreso. En este sentido, la interpretación de la radiografía de tórax puede ser importante para el correcto diagnóstico y ubicación de estos pacientes. En urgencias, la interpretación de la radiografía de tórax se realiza por el médico que atiende a ese enfermo. Una lectura radiológica errónea puede dar lugar a una ubicación inadecuada del paciente o a un incremento en la estancia media (EM).

Los objetivos de este trabajo son determinar la concordancia, entre los médicos de urgencias, en función de su experiencia, y un radiólogo, en la interpretación de la radiografía de tórax de los pacientes ingresados en una UEC y evaluar si las interpretaciones no concordantes supusieron un aumento de la EM de estos pacientes, así como determinar si existieron diferencias en el destino final de los pacientes según la concordancia radiológica.

\section{MATERIAL Y MÉTODO}

Se revisaron las historias clínicas de todos los enfermos ingresados en una UEC durante un periodo de 4 meses (diciembre de 2003 a marzo de 2004). Se recogieron datos de edad, sexo, interpretación de la radiografía por el médico de urgencias y por el radiólogo, diagnóstico de urgencias, diagnóstico al alta, estancia media y destino del paciente. Se comparó la interpretación de la radiografía de tórax del médico de urgencias responsable del paciente con la de dos radiólogos, determinando si existían diferencias según el médico de urgencias fuera un residente menor (primer o segundo año) o un residente mayor (tercer, cuarto o quinto año) o un adjunto, y si la lectura se realizó en horario diurno (definido desde las 9 horas de la mañana hasta las 21 horas) o en horario nocturno.

En los casos de no concordancia se evaluó el diagnóstico de urgencias con el diagnóstico al alta, determinando si existieron cambios de diagnóstico o de tratamiento y si la no concordancia determinó un aumento de la estancia media. Para facilitar la concordancia diagnóstica se agruparon en 6 grupos: respiratorio crónico, respiratorio agudo, cardiópata, respiratorio crónico más cardiópata, respiratorio agudo más cardiópata y otros.

El destino de los enfermos al alta de la UEC se consideró como: alta a domicilio, traslado a otro servicio de agudos, traslado a centro sociosanitario, traslado a la Unidad de Curas Paliativas y exitus.

Se definió concordancia como la coincidencia exacta en la interpretación de la radiografía de tórax. La interpretación radiológica se agrupó en tres categorías: alteración aguda (condensación nueva, signos de fallo cardiaco, derrame pleural), alteración crónica (enfermedades crónicas con cambios radiológicos como, por ejemplo, las secuelas post-tuberculosas o la presencia de alteraciones sugestivas de bronquiectasias) y hallazgos negativos o normalidad. Además, el radiólogo valoró las características técnicas de la radiografía para valorar si ésta era válida para realizar una interpretación radiológica.

El estudio estadístico se realizó con un paquete SPSS para windows. Para la comparación de medias se realizó una prueba de la t de Student, y para la comparación de proporciones se utilizó la prueba de la ji al cuadrado con corrección de Fisher en caso necesario.

\section{RESULTADOS}

Durante el periodo de estudio ingresaron en la UEC un total de 316 pacientes. Se eliminaron del estudio aquellos a los que no se realizó radiografía de tórax previa al ingreso en planta y en los que no constaba la interpretación del médico de urgencias en la hoja de ingreso. Finalmente se evaluaron 260 pacientes (118 varones y 142 mujeres), con una edad media de $77,17(13,4)$ años. La concordancia total se obtuvo en 193 pacientes $(74,2 \%)$, mientras que en $67(25,8 \%)$ no hubo concordancia. En estos 67 casos se incluyen 11 en los que el radiólogo consideró que la radiografía no tenía las características técnicas adecuadas para ser legible. El grado de concordancia según el médico que realizó la lectura fue del $66,4 \%$ si era un residente menor y del $82 \%$ si era un residente mayor o un adjunto ( $p=0,003$ ) (Fig. 1). La concordancia durante el horario diurno fue del 74,4\% y del 70,2\% durante el horario nocturno $(\mathrm{p}=\mathrm{ns})$.

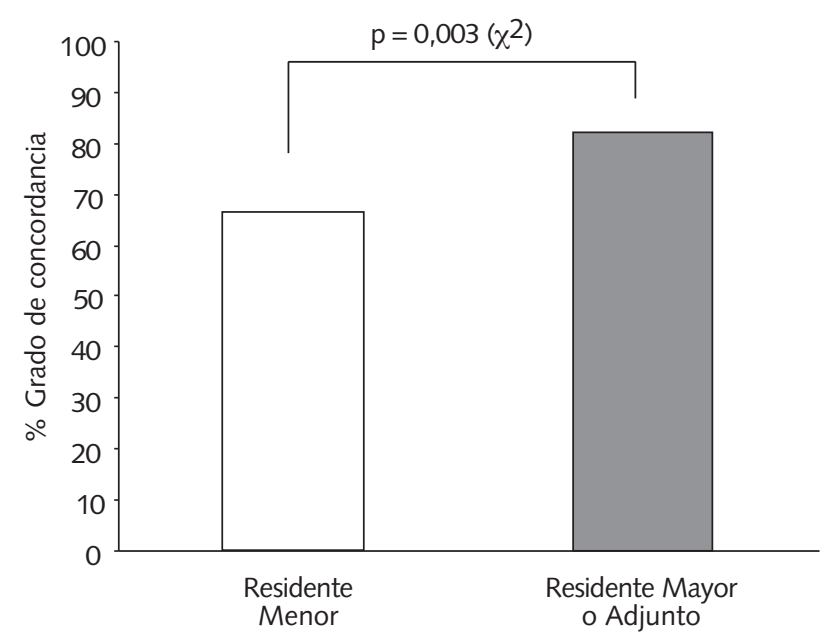

Fig. 1. Concordancia entre el médico de urgencias y el radiólogo. Existió mayor concordancia cuando el médico de urgencias era un adjunto o un residente mayor que cuando era un residente menor.

En los 67 casos en los que no hubo concordancia, solo en $9(3,5 \%)$ se hubiera realizado un cambio de diagnóstico y/o tratamiento. Los grupos diagnósticos de los pacientes se exponen en la tabla I. Los diagnósticos de urgencias y al alta del paciente de estos nueve casos se exponen en la tabla II.

El destino de los pacientes al alta de la UEC, así como el destino en el caso de no concordancia se expone en la tabla III. No se evidenciaron diferencias en el destino de los pacientes según la concordancia radiológica ni entre los casos de no concordancia según se realizó cambio de diagnóstico y/o tratamiento.

La EM global fue de 4,79 $(2,4)$ días. En aquellos pacientes en los que hubo concordancia la EM fue de 4,63 $(2,4)$ días, y cuando no había concordancia, la EM fue de 5,09 $(2,4)$ días ( $\mathrm{p}=\mathrm{ns})$. La EM de los pacientes que tuvieron un cambio diagnóstico y/o tratamiento fue de 5,89 (2,6) días ( $\mathrm{p}=0,09)$ (Fig. 2). Cuando se analizó la EM según el médico que interpretó la radiografía de tórax, se observó que cuando la interpretación la realizó un residente menor, la EM fue de 4,98 $(2,4)$ días, mien- 
TABLA I

\begin{tabular}{lc}
\multicolumn{3}{c}{ TABLA I } \\
\multicolumn{2}{c}{ CATEGORÍA DIAGNÓSTICA DE LOS PACIENTES } \\
AL ALTA DE LA UEC & Número \\
\hline \multicolumn{2}{c}{108} \\
\hline Respiratorio crónico & 53 \\
Respiratorio agudo & 34 \\
Cardiopatía & 15 \\
Respiratorio crónico más cardiopatía & 23 \\
Respiratorio agudo más cardiopatía & 27 \\
Otros & 27 \\
\hline
\end{tabular}

\section{TABLA II}

DIAGNÓSTICOS DE URGENCIAS Y AL ALTA EN LOS NUEVE PACIENTES EN LOS QUE NO HUBO CONCORDANCIA Y SE CAMBIÓ EL DIAGNÓSTICO Y/O TRATAMIENTO

\begin{tabular}{lll}
\hline & Diagnóstico de urgencias & Diagnóstico al alta \\
\hline Paciente 1 & Neumonitis eosinofilica & ICl. Asma bronquial \\
Paciente 2 & Insuficiencia cardiaca & BONO \\
Paciente 3 & Insuficiencia cardiaca & Infección bronquial. ACA \\
Paciente 4 & Neumonía EH. EPOC & Infección bronquial. EPOC \\
Paciente 5 & IC. Insuficiencia respiratoria. & Infección bronquial. EPOC \\
Paciente 6 & Neumonía EH. EPOC & Infección bronquial. ICI. EPOC \\
Paciente 7 & Insuficiencia respiratoria aguda & ICI \\
Paciente 8 & Insuficiencia respiratoria. & Infección bronquial. ICBV \\
Paciente 9 & Bronquitis aguda & Insuficiencia respiratoria. EPOC \\
\hline
\end{tabular}

$\mathrm{ICI}$ : Insuficiencia cardiaca izquierda; BONO: Bronquiolitis obliterante con neumonía organizada; AGA: Agudización grave del asma. Neumonía EH: Neumonía extrahospitalaria; EPOC: Enfermedad pulmonar obstructiva crónica; ICBV: Insuficiencia cardiaca biventricular.

\section{TABLA III}

\section{DESTINO DE LOS PACIENTES AL ALTA EN LA UEC}

\begin{tabular}{lcccc}
\hline & Totales & Concocordantes & $\begin{array}{c}\text { NC sin cambio } \\
\text { de dgt/tto }\end{array}$ & $\begin{array}{c}\text { NC con cambio } \\
\text { de dgt/tto }\end{array}$ \\
\hline Domicilio & 238 & 177 & 54 & 7 \\
Centro Soc. & 6 & 5 & 1 & 0 \\
Otro Servicio & 10 & 7 & 1 & 2 \\
UCP & 4 & 2 & 2 & 0 \\
Exitus & 2 & 2 & 0 & 0 \\
\hline
\end{tabular}

Centro Soc.: Centro sociosanitario; $p$ no significativa en el destino final de los pacientes entre concordantes y no concordantes, ni entre no concordantes con y sin cambio de diagnóstico y/o tratamiento.

tras que ésta era de 4,63 $(2,5)$ días cuando era un residente mayor o un adjunto $(\mathrm{p}=\mathrm{ns})$. Tampoco se produjeron cambios significativos en la EM según existiera concordancia o no $(4,80$ $(2,3)$ vs 4,60 $(2,54)$ cuando existía concordancia con el radiólogo y $5,31(2,5)$ vs $4,79(2,3)$ cuando no existía; $\mathrm{p}=\mathrm{ns})$.

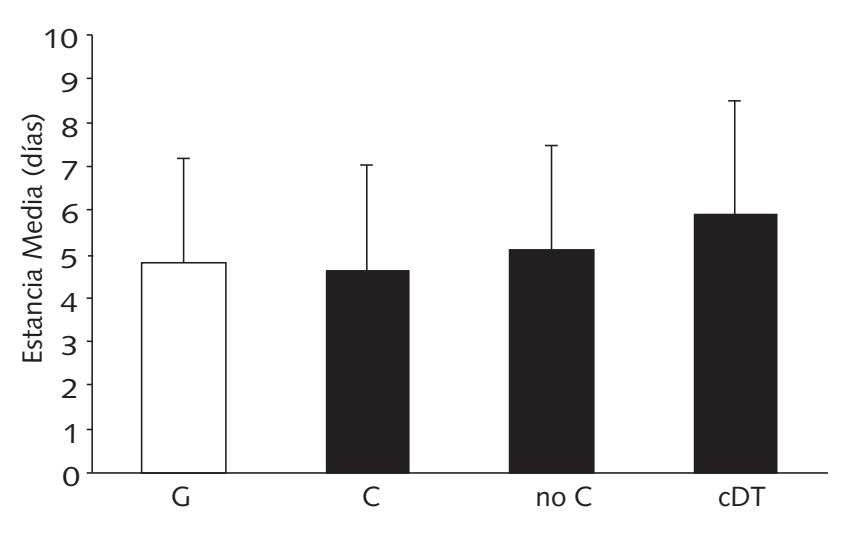

Fig. 2. Estancia media de los pacientes ingresados en la UEC según la concordancia; G: global; C: Concordancia; No C: No concordancia; CDT: Casos de no concordancia en los que se hubiera realizado cambio de diagnóstico y/o tratamiento; $p=0,09$ entre la estancia media global y el grupo CDT.

\section{DISCUSIÓN}

Las unidades de estancia corta se crearon como una alternativa a la hospitalización convencional, con el objetivo de disminuir los ingresos en las unidades hospitalizadas. Los pacientes que ingresan en una UEC deben cumplir una serie de requisitos como son la de estar afectos de una patología bien definida, no precisar la realización de maniobras diagnóstico-terapéuticas complejas y preveerse una estancia media de 4-5 días. Con estas condiciones, se ha podido objetivar que las UEC disminuyen el número de ingresos en unidades especializadas (4), así como disminuir y minimizar las causas de estancias inadecuadas $(4,5)$.

El diagnóstico realizado en los Servicios de Urgencias depende en gran parte de la interpretación correcta de la radiografía de tórax, puesto que un error en su lectura puede suponer un diagnóstico incorrecto, y por tanto, una ubicación inadecuada del paciente. De hecho, una mala interpretación de la radiografía de tórax fue la segunda causa, tras una inadecuada valoración clínica, en los casos de error diagnóstico de los pacientes en urgencias (6). Este hecho tiene aún más importancia cuando la unidad en la que se ingresa al paciente es una UEC, ya que un error en la ubicación puede suponer un incremento en las EM, y por tanto, disminuir la eficiencia de estas unidades. Es por ello que se planteó si la lectura previa de la radiografía de tórax por un radiólogo hubiera podido influir en la EM.

Nuestros resultados indican que la interpretación de la radiografía de tórax por un radiólogo previa a la decisión de ingresar a un paciente en la UEC tiene una cierta tendencia a mejorar la EM, aunque no se obtuvieron diferencias significativas. Solo en los pacientes en los que se hubiera producido un cambio de diagnóstico o tratamiento cobraría una mayor importancia, pero éstos representan sólo un pequeño porcentaje del total de pacientes ingresados. Estos resultados coinciden con un estudio previo realizado en nuestro medio en el que se evaluó el rendimiento diagnóstico de la radiografía de tórax cuando ésta era valorada por un radiólogo en pacientes que ingresaban en un servicio de Medicina Interna (7). En el estudio de Urrutia et al (7), la discordancia entre 
los médicos de urgencias y el radiólogo fue del $13.7 \%$, mientras que en nuestro estudio es del $25,8 \%$, pero los casos en los que esta discordancia hubiera obligado a un cambio de diagnóstico y/o tratamiento es similar en ambos estudios (3,8\% en el trabajo de Urrutia et al. y 3,46\% en nuestro trabajo), y no difiere de los resultados obtenidos en trabajos fuera de nuestro ámbito $(8,9)$. La diferencia en el porcentaje de discordancia entre los dos trabajos podría explicarse por el hecho de que en nuestro trabajo, cuando se informaba una radiografía como normal o sin patología aguda y existían cambios crónicos que eran valorados como tales por el radiólogo, se interpretaba como no concordante. El mayor porcentaje de pacientes crónicos ingresados en nuestra UEC justifica el incremento de casos no concordantes de nuestro estudio.

Por otro lado, la EM no varió significativamente según el médico que hubiera realizado la interpretación de la radiografía de tórax, aunque se observó una tendencia al incremento de la misma en los casos en los que no había

\section{Bibliografía}

1. Corbella X, Salazar A, Maiques JM, Juan A. Unidad de corta estancia de urgencias como alternativa a la hospitalización convencional. Med Clin (Barc) 2002; 118: 515.

2. De la Iglesia F, Ramos V, Pellicer C, Nicolas R, Diz-Lois F. La unidad de corta estancia médica (UCEM) de A Coruña: cumplimos cinco años. An Med Intern (Madrid) 2001; 18: 166.

3. Abenhaim HA, Khan SR, Raffoul J, Becker MR. Program description: a hospitalist-run, medical short-stay unit in a teaching hospital. Can Med Assoc J 2000; 163: 1477-80.

4. Márquez MA, Gutiérrez J, Supervía A, Robles MJ, Arellano M, Puig J. Unidad de estancia corta: una forma de optimizar recursos hospitalarios. Rev Calidad Asistencial 2003; 18: 315.

5. Villalta J, Sisó A, Cereijo AC, Sequeira E, de la Sierra A. Adecuación de la hospitalización en una unidad de estancia corta de un hospital concordancia con el radiólogo y la lectura la había efectuado un residente menor, llegando a aumentarse hasta los 5,31 (2,46 días).

El grado de concordancia no estuvo influido por el horario en el que se realizó la lectura, ni influyó en el destino final de los pacientes. En cambio, si que se produjo un mayor nivel de concordancia cuando la lectura la efectuó un residente mayor $(\mathrm{p}=0,003)$.

Por tanto, creemos que la interpretación radiológica previa por un radiólogo en los pacientes con previsión de ingreso en una UEC no está justificada, sobre todo si tenemos en cuenta que ello representaría un aumento de la presión, ya de por sí elevada, de los Servicios de Radiología. Si podría realizarse cuando el residente mayor o el adjunto de guardia tuvieran dudas en la interpretación de la radiografía de tórax. Por otro lado, la interpretación de la radiografía de tórax por médicos noveles exige una mayor supervisión, al igual que su actividad asistencial, para así poder mejorar el rendimiento diagnóstico en esta exploración complementaria. universitario. Un estudio controlado. Med Clin (Barc) 2004; 122: 454-6.

6. Tudela P, Mòdol JM, Veny A, Tor J, Bonet M, Rego MJ. Estudio de la concordancia diagnóstica entre el área médica de urgencias y la de hospitalización en un hospital general. Med Clin (Barc) 2002; 119: 531-3.

7. Urrutia A, Bechini J, Tor J, Olazábal A, Rey-Joli C. Evaluación de la interpretación de la radiografía de tórax por los médicos de guardia en un hospital universitario. Med Clin (Barc) 2001; 117: 332-3.

8. Espinosa JA, Nolan TW. Reducing errors by emergency physicians in interpreting radiographs: longitudinal study. Br Med J 2000; 320: 737-40.

9. Mayhue FE, Rust DD, Aldag JC, Jenkins AM, Ruthman JC. Accurancy of interpretations of emergency department radiographs: effect of confidencial levels. Ann Emerg Med 1989; 18: 826-30. 